Original

\title{
Resumen ejecutivo del Documento de Consenso de la Sociedad Española de Enfermedades Infecciosas y Microbiología Clínica (SEIMC) y de la Asociación Española de Cirujanos (AEC) en profilaxis antibiótica en cirugía
}

M. Dolores del Toro López ${ }^{a, *}$, Javier Arias Díaz ${ }^{b}$, José M. Balibrea ${ }^{c}$, Natividad Benito ${ }^{d}$, Andrés Canut Blasco ${ }^{e}$, Erika Esteve ${ }^{f}$, Juan Pablo Horcajada ${ }^{f}$, Juan Diego Ruiz Mesa ${ }^{g}$, Alba Manuel Vázquez ${ }^{h}$, Cristóbal Muñoz Casares ${ }^{i}$, Jose Luis del Pozo ${ }^{j}$, Miquel Pujol ${ }^{k}$, Melchor Riera ${ }^{l}$, Jaime Jimeno ${ }^{m}$, Inés Rubio Pérez ${ }^{n}$, Jaime Ruiz-Tovar Polo ${ }^{\circ}$, Alejandro Serrablo ${ }^{p}$, Alex Soriano ${ }^{q}$, Josep M. Badia ${ }^{r}$ y Grupo de Estudio de PA de la Sociedad Española de Enfermedades Infecciosas y Microbiología Clínica (SEIMC) y Asociación Española de Cirujanos (AEC)

${ }^{a}$ Unidad Clínica de Enfermedades Infecciosas, Microbiología y Medicina Preventiva, Hospital Universitario Virgen Macarena, Universidad de Sevilla, IBIS, Sevilla, España

b Servicio de Cirugía, Hospital Clínico San Carlos, y Departamento de Cirugía, Facultad de Medicina, Universidad Complutense de Madrid, Madrid, España

' Servicio de Cirugía Gastrointestinal ICMDM, Hospital Clínic de Barcelona, Universitat de Barcelona, Barcelona, España

${ }^{\mathrm{d}}$ Unidad de Enfermedades Infecciosas, Hospital de la Santa Creu i Sant Pau, Institut d'Investigació Biomèdica Sant Pau,

Universitat Autònoma de Barcelona, Barcelona, España

e Servicio de Microbiología, Hospital Universitario de Álava, Vitoria-Gasteiz, Álava, España

${ }^{\mathrm{f}}$ Servicio de Enfermedades Infecciosas, Hospital del Mar, IMIM, Barcelona, España

${ }^{\mathrm{g}}$ Servicio de Enfermedades Infecciosas, Hospital Regional Universitario de Málaga, IBIMA, Málaga, España

${ }^{\mathrm{h}}$ Servicio de Cirugía General, Hospital Universitario de Guadalajara, Guadalajara, España

${ }^{\text {i }}$ Servicio de Cirugía General, Hospital Universitario Virgen del Rocío, Sevilla, España

${ }^{j}$ Servicio de Enfermedades Infecciosas y Microbiología, Clínica Universidad de Navarra, Universidad de Navarra, Pamplona, Navarra, España

${ }^{\mathrm{k}}$ Servicio de Enfermedades Infecciosas, Hospital Universitari de Bellvitge, L'Hospitalet de Llobregat, Barcelona, España

${ }^{1}$ Servicio de Enfermedades Infecciosas, Hospital Son Espases, Palma, Mallorca, España

${ }^{\mathrm{m}}$ Servicio de Cirugía General, Hospital Universitario Marqués de Valdecilla, Santander, Cantabria, España

${ }^{\mathrm{n}}$ Servicio de Cirugía General, Hospital Universitario La Paz, Madrid, España

- Servicio de Cirugía General, Hospital Universitario Rey Juan Carlos, Móstoles, Madrid, España

${ }^{\mathrm{p}}$ Servicio de Cirugía General, Hospital Universitario Miguel Servet, Zaragoza, España

${ }^{\mathrm{q}}$ Servicio de Enfermedades Infecciosas, Hospital Clínic de Barcelona, IDIBAPS, Universitat de Barcelona, Barcelona, España

${ }^{\mathrm{r}}$ Servicio de Cirugía General, Hospital General de Granollers, Universitat Internacional de Catalunya, Granollers, Barcelona, España

Este artículo se publica simultáneamente en: Enfermedades Infecciosas y Microbiología Clínica:10.1016/j.eimc.2020.02.017, con el consentimiento de los autores y editores.

* Autor para correspondencia.

Correo electrónico: mdeltoro@us.es (M.D. del Toro López).

https://doi.org/10.1016/j.ciresp.2020.03.022

0009-739X/C 2020 Publicado por Elsevier España, S.L.U. en nombre de AEC. 


\section{INFORMACIÓN DEL ARTÍCULO}

\section{Historia del artículo:}

Recibido el 18 de febrero de 2020

Aceptado el 1 de marzo de 2020

On-line el $\mathrm{xxx}$

Palabras clave:

Profilaxis antibiótica

Infección de localización quirúrgica

Prevención de la infección

Procedimientos quirúrgicos

\section{R E S U M E N}

La profilaxis antibiótica en cirugía es una de las medidas más eficaces para la prevención de la infección de localización quirúrgica, aunque su uso es con frecuencia inadecuado, pudiendo incrementar el riesgo de infección, toxicidades y resistencias bacterianas. Debido al avance en las técnicas quirúrgicas y la emergencia de microorganismos multirresistentes las actuales pautas de profilaxis precisan ser revisadas.

La Sociedad Española de Enfermedades Infecciosas (SEIMC), conjuntamente con la Asociación Española de Cirujanos (AEC) ha revisado y actualizado las recomendaciones de profilaxis antimicrobiana para adaptarlas a cada tipo de intervención quirúrgica y a la epidemiología actual. En este documento se recogen las recomendaciones de los antimicrobianos utilizados en profilaxis en los diferentes procedimientos, las dosis, la duración, la profilaxis en huéspedes especiales, y en situación epidemiológica de multirresistencia, de tal forma que permitan un manejo estandarizado, un uso racional, seguro y efectivo de los mismos en la cirugía electiva.

(C) 2020 Publicado por Elsevier España, S.L.U. en nombre de AEC.

\section{Executive summary of the Consensus Document of the Spanish Society of Infectious Diseases and Clinical Microbiology (SEIMC) and of the Spanish Association of Surgeons (AEC) in antibiotic prophylaxis in surgery}

\section{A B S T R A C T}

Antibiotic prophylaxis in surgery is one of the most effective measures for preventing surgical site infection, although its use is frequently inadequate and may even increase the risk of infection, toxicities and antimicrobial resistance. As a result of advances in surgical techniques and the emergence of multidrug-resistant organisms, the current guidelines for prophylaxis need to be revised.

The Sociedad Española de Enfermedades Infecciosas (Spanish Society of Infectious Diseases and Clinical Microbiology) (SEIMC) together with the Asociación Española de Cirujanos (Spanish Association of Surgeons) (AEC) have revised and updated the recommendations for antibiotic prophylaxis in surgery to adapt them to any type of surgical intervention and to current epidemiology. This document gathers together the recommendations on antimicrobial prophylaxis in the various procedures, with doses, duration, prophylaxis in special patient groups, and in epidemiological settings of multidrug resistance to facilitate standardized management and the safe, effective and rational use of antibiotics in elective surgery.

(C) 2020 Published by Elsevier España, S.L.U. on behalf of AEC.

\section{Introducción}

\section{Necesidad del documento y objetivos}

La profilaxis antibiótica (PA) en cirugía es una de las medidas más eficaces para la prevención de la infección de localización quirúrgica (ILQ). A pesar de ello, su uso es con frecuencia inadecuado, en general porque no se administra según las guías, no se inicia en el momento adecuado o porque se prolonga demasiado. Esta administración inadecuada puede incrementar el riesgo de ILQ de toxicidades y de resistencias bacterianas, cuyo control es un objetivo prioritario de las autoridades sanitarias.
El avance y la aparición de nuevas técnicas quirúrgicas, el incremento del número de trasplantes, y la emergencia y expansión de microorganismos multirresistentes, obliga a revisar las pautas de PA utilizada en décadas anteriores.

El último documento de consenso español de PA se publicó en 2002. La Sociedad Española de Enfermedades Infecciosas (SEIMC), conjuntamente con la Asociación Española de Cirujanos (AEC) ha revisado y actualizado las recomendaciones de PA para adaptarlas a cada tipo de intervención quirúrgica y a la epidemiología actual. Las recomendaciones realizadas en este documento están basadas en la evidencia científica. Cuando no ha sido posible encontrar evidencia de calidad los coordinadores y autores del documento han dado una recomendación basada en conocimientos de etiopatogenia y factores de riesgos de ILQ en estudios farmacocinéticos 
de los antibióticos utilizados en profilaxis y en la experiencia clínica.

Este documento de consenso pretende proporcionar unas directrices que permitan un manejo estandarizado de la PA en cirugía electiva, con un uso racional, seguro y efectivo de los antimicrobianos para la prevención de las ILQ.

\section{Alcance y ámbito del documento}

Este documento se centra exclusivamente en la PA quirúrgica y no abarca otras medidas que se han demostrado eficaces en la prevención de la ILQ como la descolonización de Staphylococcus aureus o la antisepsia de la piel. Se dan unas recomendaciones generales de PA e indicaciones específicas por tipos de procedimientos quirúrgicos, con grados de recomendación según la evidencia científica. Se facilitan los antimicrobianos y las dosis recomendadas, aunque se excluyen algunos que el comité ha considerado no adecuados para la profilaxis por la elevada tasa de resistencias actuales, excesivo espectro, impacto ecológico o capacidad de inducir resistencias (p. ej., quinolonas o ertapenem). También se hacen recomendaciones acerca de la duración, la profilaxis en huéspedes especiales y en situación epidemiológica de multirresistencia.

Una de las limitaciones más importantes de este documento es que no siempre se pueden dar recomendaciones con alto grado de evidencia debido al diseño de la mayoría de los trabajos, la escasez de estudios comparativos con placebo o diferentes antimicrobianos que estudien la eficacia de la PA y a la baja incidencia de infecciones en la mayoría de los procedimientos. En ocasiones se dan recomendaciones por inferencia de la evidencia en otras cirugías en la misma región anatómica y con espectro microbiano similar. Aunque se recomiendan determinados tipos de antibióticos, su elección debe adaptarse a la epidemiología local y a los programas de optimización del uso de antimicrobianos en cada centro. No es posible hacer indicaciones generales en situaciones complejas, como pacientes sometidos a múltiples cirugías y que han recibido diversos antimicrobianos, donde habrá que individualizar la profilaxis dependiendo del riesgo de infección y del estado de colonización del paciente.

El documento está dirigido a los profesionales de atención especializada implicados en el procedimiento quirúrgico, como anestesistas y cirujanos, y en aquellos que participan en la prevención de la infección quirúrgica, como infectólogos, microbiólogos, preventivistas y farmacéuticos.

\section{Metodología}

Las 2 sociedades nombraron coordinadores para este proyecto, un infectólogo (MDT) y un cirujano (JMB), que seleccionaron al resto del panel de expertos, que incluye cirujanos, infectólogos, internistas y microbiólogos. El manuscrito final estuvo a disposición de los miembros de ambas sociedades para su revisión y sugerencias.

El documento es una actualización del publicado en 2002, basado en guías publicadas recientemente en las que se contestan a preguntas de interés clínico.

Con el fin de responder a cada pregunta, se realizó una búsqueda sistemática bibliográfica (Biblioteca Cochrane Plus [Cochrane Library], Medline, EMBASE, Scopus, Tryp database,
DARE), desde 1970 hasta octubre de 2018, aunque se incluyen algunos trabajos que se publicaron durante la revisión del documento y que se consideraron relevantes. Los términos de búsqueda se especifican en cada apartado del documento original.

De acuerdo con la normativa de la SEIMC se utilizaron los criterios establecidos por la SEIMC para la gradación de la evidencia (tabla 2 del documento original) y la evaluación de la calidad metodológica de acuerdo con la Agree Colaboration (http://www.agreecollaboration.org).

El texto completo fue revisado y aprobado por todos los autores, y estuvo abierto en la página web de la SEIMC para su revisión por todos los socios. Los conflictos de intereses de todos los miembros del panel de expertos se especifican al final del documento.

\section{Recomendaciones}

\section{Principios básicos de la profilaxis en cirugía}

¿Cuándo está indicada la profilaxis?

La PA en cirugía está recomendada:

- Cuando las probabilidades de infección sean altas o cuando las consecuencias de una infección postoperatoria sean potencialmente graves para el paciente (endocarditis, endoftalmitis, infección protésica) (A-III).

- En las cirugías clasificadas como limpia-contaminada y contaminada (A-II).

- En la cirugía sucia, en la que existe supuración o infección evidentes, los antibióticos se administran en forma de tratamiento (A-III).

- En la cirugía limpia, la indicación de la PA depende del tipo de intervención, de la comorbilidad del paciente y de la existencia de material protésico, aunque en algunos casos no ha sido aun totalmente esclarecida.

\section{¿Cuál es el antimicrobiano más adecuado?}

Los antibióticos deben ser activos frente a los microorganismos aislados con más frecuencia en cada tipo de procedimiento, aunque la mayor parte de expertos aconsejan la utilización de una cefalosporina de primera o segunda generación (A-III). Los microorganismos involucrados en las ILQ por tipo de cirugía en 13 países europeos se muestran en la tabla 3 del documento original.

- La elección de los antibióticos debe tener en cuenta la epidemiología local y los patrones de resistencia a los antimicrobianos de los microorganismos que causan las infecciones quirúrgicas en el hospital (A-III).

- Las cefalosporinas de $1 .^{a}$ y $2 .^{a}$ generación, pero fundamentalmente cefazolina, son los antibióticos de elección en la mayoría de las indicaciones (A-I).

- En caso de alergia a betalactámicos, antecedentes de colonización o infección por Staphylococcus aureus resistente a meticilina (SARM), o prevalencia elevada en el hospital de ILQ por este microorganismo, puede emplearse un glucopéptido (A-I). 
- En cirugía de colon o ginecológica, donde la implicación de los microorganismos anaerobios y enterobacterias en la colonización del sitio quirúrgico es esperable, se aconseja elegir un antibiótico o combinación de antibióticos con actividad frente a ambos grupos de microorganismos (A-I).

¿Cuál es el momento óptimo para administrar la profilaxis?

- La PA en cirugía debe ser administrada durante los 120 min previos a la incisión quirúrgica (A-I).

- En el caso de los betalactámicos de semivida corta (p. ej., penicilinas y cefalosporinas como cefazolina, cefoxitina y cefuroxima) es aconsejable su administración durante los 60 min previos a la incisión quirúrgica (B-II).

- En el caso de vancomicina, aminoglucósidos o fluoroquinolonas, la infusión intravenosa debe comenzar $90 \mathrm{~min}$ antes de la incisión quirúrgica, ya que estos antibióticos requieren periodos de infusión largos (B-II).

- En el caso de cirugía que requiera la isquemia del miembro, administrar la profilaxis antes de hinchar el torniquete (A-III).

¿Cuál es la dosis adecuada?

Se acepta de forma general que la dosis de un antibiótico para profilaxis es la misma que la utilizada para el tratamiento de la infección (A-III). La dosis inicial de los antimicrobianos en la profilaxis quirúrgica tanto en adultos como en niños, oral e intravenosa se recogen en las tablas 4 y 5 del documento original.

¿Hay que modificar la dosis en el paciente obeso?

- En los pacientes obesos, las concentraciones de algunos antibióticos pueden modificarse debido a alteraciones farmacocinéticas. Parámetros farmacocinéticos como el volumen de distribución y el aclaramiento del fármaco pueden ser mayores en los pacientes obesos, pero a menudo no son proporcionales al peso corporal total (A-II).

- Los pacientes obesos pueden requerir dosis iniciales más elevadas. Las dosis convencionales para pacientes no obesos pueden conducir a la infradosificación, mientras que las dosificaciones basadas en el peso corporal total tienden a la sobredosificación en los obesos (A-II).

- La utilización de descriptores subrogados del peso corporal total, como el peso ideal o el peso ajustado pueden corregir las sobredosificaciones en obesos basadas en el peso total (A-II).

- El cálculo de las dosis de mantenimiento en una profilaxis prolongada (p. ej., cirugía de larga duración) en los obesos no está bien resuelto, aunque la aproximación a la selección de dosis basadas en la estimación de la función renal puede ser una alternativa razonable y clínicamente útil (A-II).

\section{¿Es necesario repetir la dosis durante la cirugía?}

- Se recomienda una dosis adicional intraoperatoria cuando el procedimiento quirúrgico exceda 2 veces la semivida del antibiótico (B-II).
- Con cefazolina u otro antibiótico con semivida similar se debe administrar una segunda dosis intraoperatoria a las $3 \mathrm{~h}$ (B-II).

- Se recomienda una dosis adicional cuando la semivida del antibiótico está disminuida (quemados, elevadas tasas de filtración glomerular) o sangrado significativo (> $1.500 \mathrm{ml}$ en adultos o $25 \mathrm{ml} / \mathrm{kg}$ en niños) (B-II).

¿Cuál es la duración más adecuada?

- Para la mayoría de procedimientos quirúrgicos, una dosis única de un antibiótico cuya semivida asegure niveles de fármaco en suero y tejido suficientes mientras dura la intervención quirúrgica es adecuada (A-I).

\section{¿Cuáles son los efectos adversos de la profilaxis antibiótica en cirugía?}

Diarrea por Clostridioides difficile

- Existe un mayor riesgo de infección por C. difficile con algunos antibióticos utilizados en PA, como las cefalosporinas, los carbapenemas, las fluoroquinolonas o la clindamicina (A-II).

- El riesgo de infección por C. difficile es mayor si se prolonga la duración de la PA (A-II).

\section{Incremento de fracaso renal agudo}

- Los procedimientos de cirugía mayor y traumatológica pueden exponer a los pacientes en el periodo perioperatorio a fracaso renal agudo inespecífico, aún sin enfermedad renal previa. Además, estos pacientes pueden recibir profilaxis con antimicrobianos como los aminoglucósidos o glucopéptidos, que se asocian a nefrotoxicidad (A-II).

- Se deben solicitar mediciones secuenciales de creatinina sérica y urinaria, tanto en el preoperatorio como $\geq 24 \mathrm{~h}$ después de la cirugía, a los pacientes de cirugía mayor para comprobar el grado de función renal, con especial atención a los pacientes que hayan recibido profilaxis con aminoglucósidos o glucopéptidos (A-II).

\section{Influencia sobre la resistencia a los antibióticos}

- La utilización de unidosis en profilaxis quirúrgica, con las excepciones especiales (como cirugía prolongada y pérdida de sangre significativa, entre otras), contribuye a minimizar la resistencia adquirida a los antimicrobianos (A-II).

¿Qué debemos hacer ante un paciente que refiera alergia a los betalactámicos?

- La clarificación de la alergia a betalactámicos debe formar parte de la rutina de la consulta de anestesia y de los cuidados del preoperatorio (B-II). 
- Los pacientes con historia de reacción alérgica grave tanto inmediata, de inicio en la primera hora tras la administración de un betalactámico, como no inmediata, no deben recibir profilaxis con betalactámicos, cuando haya otras alternativas terapéuticas eficaces (B-III).

- Se podrían emplear otros betalactámicos (con cadena lateral diferente al betalactámico implicado en la reacción alérgica) siempre y cuando el estudio alergológico lo haya corroborado mediante pruebas de exposición (B-II).

- Las guías locales de PA deben contemplar fármacos alternativos a los betalactámicos, de probada eficacia, para estos pacientes alérgicos (B-III).

¿Hay que cambiar la profilaxis en los pacientes colonizados por microorganismos multirresistentes?

- En cirugía de alto riesgo (cardiaca, ortopédica) en pacientes colonizados por SARM, la profilaxis puede realizarse con un glucopéptido más un betalactámico, acompañado del resto de medidas para la descolonización (A-II).

- En pacientes colonizados por enterobacterias productoras de betalactamasas de espectro extendido (BLEE), su cobertura en profilaxis debe considerarse solo en pacientes de alto riesgo (B-III).

\section{Recomendaciones por tipo de cirugía}

Los antibióticos recomendados para cada tipo de procedimiento quirúrgico se muestran en la tabla 1 del presente documento y su dosificación en las tablas 4 y 5 del documento original.

\section{Cirugía limpia}

Se han descrito unos criterios generales a partir de los que se puede prescindir de la PA (tabla 6 del documento original). En cirugía limpia no protésica de duración inferior a $2 \mathrm{~h}$, sin gran atrición tisular, no es necesario emplear PA puesto que la tasa de infección debe situarse muy por debajo del 3\%. La profilaxis está indicada cuando se coloca una prótesis o un implante intravascular y cuando los efectos de la infección sean muy graves (endoftalmitis, infección de una malla o de un dispositivo de acceso vascular).

\section{Cirugía plástica y dermatológica}

La PA no está recomendada en la cirugía limpia sin implante. Está recomendada si la operación se acompaña de un implante o existen los factores de riesgo que se detallan en la tabla 6 del documento original.

\section{Cirugía de la hernia}

Existe controversia acerca de la profilaxis en cirugía herniaria. Ante la dificultad de predecir alguno de los factores de riesgo de ILQ en el período preoperatorio, se recomienda la profilaxis en herniorrafia y hernioplastia inguinal abierta y en el resto de las hernioplastias abdominales (por inferencia a partir de la evidencia en hernia inguinal).

\section{Cirugía de mama y cirugía oncoplástica}

La profilaxis es eficaz en la cirugía oncológica de mama sin reconstrucción, en la reconstrucción inmediata, en la cirugía de reducción de mama y en los implantes con fines estéticos.

\section{Cirugía cardiaca y vascular}

By-pass aortocoronario y recambio valuular. La PA en este tipo de procedimientos disminuye de manera eficaz la tasa de infección asociada. Las cefalosporinas de $1 .^{\mathrm{a}}$ y $2 .^{\mathrm{a}}$ generación son los antimicrobianos más utilizados, no hay una evidencia clara que apoye el uso de un glucopéptido como profilaxis en instituciones con una prevalencia elevada de SARM. Una dosis única de antibiótico parece suficiente si se mantienen los niveles séricos durante el procedimiento.

No existe evidencia sobre la PA antes de la implantación de válvula cardiaca por vía transaórtica o TAVI, pero parece razonable la administración de una dosis de antibiótico antes del procedimiento.

Inserción de marcapasos y desfibriladores. La PA ha demostrado su eficacia en varios estudios y en un metaanálisis.

Implantación de catéteres de acceso vascular central. No se recomienda la profilaxis antes de la inserción de catéteres vasculares centrales, ni tampoco el sellado antibiótico de manera rutinaria antes de insertar o manipular un catéter intravascular.

Cirugía vascular periférica (percutánea y abierta). La PA se recomienda en procedimientos con colocación de material protésico o en los de alto riesgo como reparación de aneurismas, trombendarterectomía o bypass venosos. Los procedimientos braquiocefálicos (endarterectomía carotídea, reparación de la arteria braquial) sin implantación de material protésico no parecen beneficiarse de la PA. Los factores de riesgo asociados a la colocación de stents son: duración prolongada $(>2 \mathrm{~h})$, reintervenciones, stents en extremidades inferiores, hematomas, portadores de otros dispositivos intravasculares e inmunosuprimidos.

\section{Cirugía oftálmica}

Se recomienda el uso de antibióticos intracamerales administrados a la conclusión de la cirugía, en cirugía de cataratas y cirugía de glaucoma/injertos corneales. En los traumatismos penetrantes oculares se recomienda la PA intravítrea. La PA también está indicada en cirugía del lacrimal.

\section{Procedimientos neuroquirúrgicos}

Craneotomía. La PA disminuye la tasa de infección tras la realización de una craneotomía, tanto en la aparición de ILQ como en el desarrollo de meningitis.

Colocación de derivación ventrículo-peritoneal (DVP) o ventrículoauricular (DVA) y drenaje ventricular externo (DVE). La PA es recomendada en la colocación de DVP, DVA y VVE. No se recomienda para la colocación de sensor de presión intracraneal. 
Tabla 1 - Recomendación de profilaxis antibiótica según el tipo de cirugía y procedimiento quirúrgico

Cirugía limpia $\quad$ Indicación Antimicrobiano recomendado

Cirugía plástica y dermatológica

No recomendada en la cirugía limpia sin implante (D-I)

Recomendada si existen factores de riesgo o implante ${ }^{a}$

(C-II)

Herniorrafia y hernioplastia inguinal Recomendada (B-I)

abierta

Hernioplastia abdominal abierta

Recomendada por inferencia a partir de la evidencia en hernia inguinal (B-II)

Hernioplastia inguinal por vía

laparoscópica

Cirugía de mama y cirugía

oncoplástica

No recomendada (D-I)

Recomendada en cirugía oncológica de mama si

factores de riesgo o neoadyuvancia (A-II)

Recomendada en cirugía de reconstrucción oncoplástica

(A-I)

Recomendada en cirugía estética (aumento, reducción)

(B-II)

Cirugía cardiaca y vascular
Cefazolina (A-II)

Cefazolina (A-I), en colonizados por SARM añadir vancomicina (B-III) Cefazolina (A-I), en colonizados por SARM añadir vancomicina (B-III)

Cefazolina (A-I)

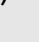

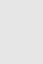

Antimicrobiano recomendado

\begin{tabular}{lc}
\multicolumn{1}{c}{$\begin{array}{c}\text { Antimicrobiano alternativo si } \\
\text { alergia a betalactámicos }\end{array}$} & Duración \\
\hline $\begin{array}{l}\text { Vancomicina/teicoplanina } \\
\text { o clindamicina (B-II) }\end{array}$ & Una dosis (A-II) \\
&
\end{tabular}

Vancomicina/teicoplanina o clindamicina (B-II)

Vancomicina/teicoplanina Una dosis (A-I)

o clindamicina (B-II)

Clindamicina o vancomicina (B-II)

Una dosis (A-I)
Bypass aortocoronario y

recambio valvular

\section{No recomendada en procedimientos cardiacos} percutáneos (D-II)

Recomendada en recambio valvular abierto y bypass

aortocoronario (A-I) y en la implantación valvular

transaórtica (B-III)

Inserción de marcapasos y

desfibriladores

Implantación de catéteres de

acceso vascular central

Recomendada (A-I)

Cefazolina o cefuroxima (A-I)

Cefazolina o cefuroxima (A-I)

No recomendada en inserción catéteres vasculares centrales (D-I)

No recomendado el sellado antibiótico de manera

rutinaria antes de insertar o manipular un catéter

intravascular (D-I)

Recomendada en un procedimiento vascular de alto riesgo incluyendo aquellos en los que se va a

implantar algún tipo de material protésico (A-I)

Cirugía vascular periférica

(percutánea y abierta)

Cefazolina (A-I)

Se sugiere añadir un segundo

antibiótico con actividad frente

a bacilos gramnegativos (gentamicina)

(B-III) si existe riesgo de exposición

a microbiota intestinal

Cirugía oftálmica

Indicación

Antimicrobiano recomendado

Cirugía de cataratas

Cirugía de glaucoma, injerto corneal

Cirugía lagrimal
Recomendada la administración intracameral (A-I)

Recomendada la administración intracameral por inferencia de la cirugía de catarata (A-II) Recomendada (A-III)

Cefuroxima o cefazolina intracameral
(A-I)
Cefuroxima intracameral (A-I)

Cefazolina o cefuroxima (A-III)
Antimicrobiano alternativo si alergia

a betalactámicos

Vancomicina (A-II)

Vancomicina (B-II) o clindamicina (C-III)

Una dosis (A-I)

\section{Una dosis (A-I)}

En caso de administrar

cefalosporinas, redosificar

$1 \mathrm{~g} /$ cada $3 \mathrm{~h}$ durante el

procedimiento, no continuar

tras el cierre (A-II)

Una dosis (A-I)

Vancomicina (A-II)

$-x$

Antimicrobiano alternativo si alergia a betalactámicos

$\begin{array}{lc}\text { Vancomicina o moxifloxacino } & \text { Una dosis (A-I) } \\ \text { intracameral (A-III) } & \\ \begin{array}{l}\text { Vancomicina o moxifloxacino } \\ \text { intracameral (B-III) }\end{array} & \text { Una dosis (A-I) } \\ \text { Vancomicina (B-III) } & \text { Una dosis (A-I) }\end{array}$




Craneotomía
Colocación de derivación ventrículo-
peritoneal o ventrículo-auricular
(DVPA) y drenaje ventricular
externo (DVE)
Colocación de sensor de presión
intracraneal (PIC)
Cirugía a través de senos
paranasales o faringe

paranasales o faringe

Cirugía de cabeza y cuello

Cirugía limpia de cabeza y cuello

No recomendada en cirugía limpia de procedimientos de cabeza y cuello (D-I) Recomendada en linfadenectomías extensas o cirugía cervical con resección multivisceral (B-II)

Cirugía limpia-contaminada de cabeza y cuello: amigdalectomía, adenoidectomía, laringuectomía, traqueotomía y cualquier otra cirugía en la que se realice incisión de la mucosa faringo-laríngea Cirugía de senos paranasales y de oído medio

Recomendada en cirugía de cabeza y cuello

limpia-contaminada (A-II), excepto en

adenoidectomía y amigdalectomía (D-I)

Recomendada en cirugía de cáncer de cabeza

y cuello (A-II)

No recomendada en la cirugía endoscópica de los senos paranasales (D-I)

No recomendada en la cirugía limpia otológica (D-I)

Recomendada la aplicación tópica de antibiótico

después de timpanoplastia (A-I)

Recomendada en la cirugía limpia contaminada y contaminada (B-III)

Recomendada en la cirugía con implante coclear (B-III)

Cirugía maxilo-facial
Antimicrobiano recomendado

\section{Cefazolina (A-I)}

Cefazolina (A-I)

En caso de alta prevalencia de SARM:

vancomicina (A-I)

Antimicrobiano alternativo si alergia a betalactámicos

Duración

Vancomicina o clindamicina (A-II)

Una dosis (A-I)

Vancomicina (A-II) Una dosis (A-I)

Amoxicilina-ácido clavulánico (A-III)

Clindamicina o vancomicina asociada a un aminoglucósido

(B-III)

Antimicrobiano alternativo si alergia a betalactámicos

Antimicrobiano recomendado

Cefazolina (B-II)

Clindamicina o vancomicina

$\leq 24$ h (A-II) (B-III)

Posiblemente una dosis sea suficiente (A-III)
Amoxicilina-ácido clavulánico (A-III)

Clindamicina más gentamicina (B-III) $\leq 24 \mathrm{~h}$ (A-II), posiblemente una dosis sea suficiente (A-III)
En la cirugía limpia contaminada y contaminada, amoxicilinaácido clavulánico (B-III) En el implante coclear: cefazolina (A-II)
En la cirugía limpia contaminada y contaminada: clindamicina más gentamicina (C-III) En el implante coclear: clindamicina o vancomicina (C-III)
Una dosis, para la cirugía limpia contaminada y contaminada (A-II) y para el implante coclear (A-I)

\section{Septoplastia}

Rinoplastia simple
No recomendada (D-I)

No recomendada (D-I) 


\section{Rinoplastia compleja (revisión,}

prótesis)

Fracturas maxilofaciales

Procedimientos odontológicos

y ortopédica

\section{Recomendada (B-II)}

Recomendada en fracturas maxilofaciales, sobre todo en la fractura mandibular que requiera reducción abierta (A-II)

Recomendada en la implantación de injerto óseo a través de la cavidad oral (B-II)

No recomendada en extracción dental en

pacientes sin factores de riesgo (D-II)

No recomendada en el implante oral ni la

endodoncia (D-II)

Indicación

Antimicrobiano recomendado

Cefazolina (B-II) o amoxicilina-ácido clavulánico (B-III)

Cefazolina (A-II) o amoxicilina-ácido clavulánico (B-III)

Amoxicilina $1 \mathrm{~g}$ oral (A-II)

ernativo si alergia

a betalactámicos

Vancomicina o clindamicina

(C-III)

Vancomicina o clindamicina (B-III)

Clindamicina (B-II) Una dosis (A-I)

Una dosis (A-I)

Una dosis (A-I)

Antimicrobiano alternativo si alergia a betalactámicos

\section{Reducción de fractura}

cerrada sin material

osteosíntesis y otra

cirugía limpia

ortopédica sin

instrumentación

No recomendada en cirugía limpia

ortopédica sin instrumentación (D-II)

Valorar en pacientes de riesgo, puede

valorarse utilizar la profilaxis en caso

de ligamentoplastia (B-III), de acuerdo

en lo recomendado en la artroplastia

Reducción de fractura cerrada con material

Recomendada (A-I)

osteosíntesis

Cirugía de fractura abierta Recomendada (A-I)

Retirada de implantes

ortopédicos utilizados

No recomendada (D-II)
Cefazolina o cefuroxima (A-I)

Si riesgo de SARM: vancomicina

o teicoplanina (B-II) más cefazolina

o cefuroxima si riesgo de ILQ por BGN.

Si riesgo de BGN resistentes, añadir gentamicina (B-III)

Cefazolina (A-I) o amoxicilina-ácido clavulánico (B-III) (fracturas grados

I y II de Gustilo)

Añadir un aminoglucósido

(gentamicina) en las fracturas grado

III de Gustilo (B-II)
Vancomicina o teicoplanina (B-II) más gentamicina si riesgo de infección por $\mathrm{BGN}$ (B-III)

Vancomicina 0

clindamicina \pm gentamicina

(B-III) $\leq 24 \mathrm{~h}$ (A-I), probablemente una dosis es suficiente (A-II)

como tratamiento

de fracturas 
Tabla 1 (Continuación)

Cirugía traumatológica y ortopédica
Indicación

Artroplastias (PTC, PTR, Recomendada (A-I)

megaprótesis

tumorales, primarias

y revisión)

Laminectomías y

discectomías $\sin /$ con

instrumentación

Recomendada en cirugía ortopédica

vertebral con/sin instrumentación,

incluyendo fusión vertebral,

laminectomía y discectomí

mínimamente invasiva (A-I)

Amputación de miembros Recomendada en la amputación de extremidades inferiores (A-II)
Antimicrobiano recomendado

Cefazolina o cefuroxima (A-I)

Cefazolina o cefuroxima (A-I)

Cefazolina o cefuroxima

o amoxicilina-ácido clavulánico (A-II)

B-II) (más gentamicina si Probablemente una dosis

riesgo de infección por BGN) sea suficiente (A-II)

(B-III)

Vancomicina o teicoplanina $\quad \leq 24 \mathrm{~h}$ (A-I)

(B-II) (más gentamicina si Probablemente una dosis

riesgo de infección por BGN) sea suficiente (A-II)

\section{(B-III)}

Vancomicina o teicoplanina Al menos $24 \mathrm{~h}$, siempre que

más gentamicina (B-III) no haya tejido infectado

remanente postamputación

(B-III)

Antimicrobiano

Duración alternativo si alergia a betalactámicos

\begin{tabular}{|c|c|c|c|c|}
\hline $\begin{array}{l}\text { Cirugía torácica mayor } \\
\text { y mínimamente } \\
\text { invasiva }\end{array}$ & $\begin{array}{l}\text { Recomendada en cirugía torácica mayor (A-I) } \\
\text { Recomendada en cirugía torácica } \\
\text { mínimamente invasiva (videotoracoscopia, } \\
\text { mediastinoscopia) (B-III) }\end{array}$ & $\begin{array}{l}\text { Cefazolina, para la cirugía torácica } \\
\text { mayor (A-I) y para la mínimamente } \\
\text { invasiva (A-II) }\end{array}$ & $\begin{array}{l}\text { Vancomicina o teicoplanina } \\
\text { (B-III) }\end{array}$ & Una dosis (A-I) \\
\hline $\begin{array}{l}\text { Colocación de tubo } \\
\text { torácico, traumatismo } \\
\text { penetrante }\end{array}$ & $\begin{array}{l}\text { No recomendada en la colocación electiva } \\
\text { de tubos torácicos (D-II) } \\
\text { Recomendada en caso de traumatismo }\end{array}$ & Cefazolina (A-I) & $\begin{array}{l}\text { Vancomicina o teicoplanina } \\
\text { (B-III) }\end{array}$ & Una dosis (A-I) \\
\hline
\end{tabular}
penetrante

Recomendada en caso de traumatismo torácico penetrante (A-I)

Cirugía esófago-gastro-

$$
\text { Indicación }
$$

Antimicrobiano recomendado

Antimicrobiano alternativo si alergia a betalactámicos

Duración

\begin{tabular}{|c|c|c|c|c|}
\hline auodenal & & & S1 alergia a betalactamicos & \\
\hline $\begin{array}{l}\text { Con rotura de mucosa } \\
\text { (esofaguectomía, gastrectomía, } \\
\text { duodeno-pancreatectomía } \\
\text { cefálica) }\end{array}$ & $\begin{array}{l}\text { Recomendada en cirugía esofágica } \\
\text { (A-II), gástrica (A-I) y gastroduodenal } \\
\text { (A-I) }\end{array}$ & Cefazolina (A-I) & $\begin{array}{l}\text { Vancomicina o clindamicina } \\
\text { más gentamicina (B-III) }\end{array}$ & Una dosis (A-I) \\
\hline $\begin{array}{l}\text { Sin rotura de mucosa (cirugía } \\
\text { de reflujo gastroesofágico, } \\
\text { achalasia, vagotomía) }\end{array}$ & $\begin{array}{l}\text { No recomendada en cirugía esófago- } \\
\text { gastro-duodenal sin rotura de } \\
\text { mucosa (D-II) } \\
\text { Recomendada en cirugía esófago- } \\
\text { gastro-duodenal sin rotura de } \\
\text { mucosa en pacientes de alto riesgo } \\
\text { (C-III) }\end{array}$ & Cefazolina (A-I) & $\begin{array}{l}\text { Vancomicina o clindamicina } \\
\text { más gentamicina (B-III) }\end{array}$ & Una dosis (A-I) \\
\hline $\begin{array}{l}\text { Implantación de PEG (gastrotomía } \\
\text { percutánea endoscópica) }\end{array}$ & $\begin{array}{l}\text { Recomendada (A-I) } \\
\text { Se recomienda una buena antisepsia } \\
\text { del lugar de implantación (A-III) }\end{array}$ & $\begin{array}{l}\text { Cefazolina o cefuroxima } \\
\text { o amoxicilina-ácido clavulánico } \\
\text { (A-II) }\end{array}$ & $\begin{array}{l}\text { Vancomicina más gentamicina } \\
\text { o clindamicina más } \\
\text { gentamicina (B-III) }\end{array}$ & Una dosis (A-I) \\
\hline
\end{tabular}


Tabla 1 (Continuación)

Cirugía esófago-gastro-

duodenal

Cirugía bariátrica

Cirugía de intestino delgado

Esplenectomía

Traumatismo penetrante

de abdomen

Apendicectomía

Cirugía colorrectal

Profilaxis antibiótica oral y preparación mecánica del colon

Cirugía hepato-biliopancreática

\section{Colecistectomía y cirugía}

biliar

Cirugía hepática dicación

Recomendada (A-II)

Recomendada en cirugía del intestino delgado sin y con obstrucción (A-III)

No recomendada en esplenectomía sin factores de riesgo (D-II)

Recomendada en esplenectomía

traumática o electiva con factores

de riesgo (B-III)

Recomendada (A-I)

Recomendada en la apendicectomía no complicada (A-I)

Recomendada (A-I)

Recomendada la preparación mecánica del colon (A-II) asociada a antibióticos orales (A-I) en cirugía colorrectal electiva

\section{Indicación}

\section{No recomendada en colecistectomía}

laparoscópica electiva de riesgo bajo-

moderado (D-I)

Recomendada en colecistectomía

abierta (A-I) o laparoscópica de alto

riesgo (A-II)

No recomendada en hepatectomía

simple (D-I)

Recomendada en hepatectomía mayor

(incluye resección biliar extrahepática)

(A-II)

sensibilidad (A.
Antimicrobiano recomendado

Cefazolina o cefuroxima o

amoxicilina-ácido clavulánico (A-II)

En la cirugía sin obstrucción, cefazolina (A-I)

En la cirugía con obstrucción,

cefazolina más metronidazol (B-II)

o amoxicilina-ácido clavulánico (B-III)

\section{Cefazolina (A-I)}

efuroxima más metronidazol (A-I) o amoxicilina-ácido clavulánico (A-II)

Antimicrobiano alternativo

si alergia a betalactámicos

Vancomicina más gentamicina

clindamicina más

gentamicina (B-III)

En la cirugía sin obstrucción,

clindamicina más gentamicina

(B-III)

En la cirugía con obstrucción

metronidazol más gentamicina (B-II)

Vancomicina más gentamicina

o clindamicina más

gentamicina (B-III)

Metronidazol más gentamicina

(A-III)

Cefuroxima más metronidazol (A-I)

o amoxicilina-ácido clavulánico (A-II)

Metronidazol más gentamicina (B-III)

Cefuroxima más metronidazol

o amoxicilina-ácido clavulánico (A-II),

añadir gentamicina en caso de alta

prevalencia de BGN resistentes (B-III)

Neomicina 1.000 mg más

metronidazol 500 mg (A-II)

Antimicrobiano recomendado

ntimicrobiano alternativo si alergia a betalactámicos

Vancomicina más gentamicina o clindamicina más gentamicina (B-III)

Cefazolina (A-I) o amoxicilina-ácido clavulánico (A-III)

Vancomicina más gentamicina (A-III)

Metronidazol más gentamicina
Duración

Una dosis (A-I)

Una dosis (A-I)

Una dosis (A-I)

Si lesión de víscera hueca, $\leq 24 \mathrm{~h}$ (A-II)

Una dosis (A-I)

Una dosis (A-I)

Tres dosis preoperatorias 19,18 y $9 \mathrm{~h}$ antes del inicio del procedimiento

Duración

$\leq 24 \mathrm{~h}$ (A-I), posiblemente una dosis sea suficiente (A-II) 
Cirugía de bajo riesgo (no manipulación

de vía biliar): cefazolina (A-I) o amoxicicilinaácido clavulánico (A-III) Añadir gentamicina en caso de alta prevalencia de BGN resistentes (B-III)

Cirugía de alto riesgo con información microbiológica de la bilis: ajustar a microbiología previa (A-II)

Cirugía de alto riesgo sin información

microbiológica de la bilis: amoxicilina-ácido clavulánico más gentamicina (B-III)

Cirugía peritoneal Recomendada en la cirugía oncológica avanzadaperitonectomía

Cirugía urológica peritoneal avanzada (A-II)

Indicación

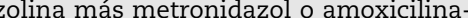

cido clavulánico (A-III)

Añadir gentamicina si riesgo de infección por BGN resistentes (B-III) a betalactámicos
Vancomicina más

gentamicina (B-II)

\section{$\leq 24 \mathrm{~h}$ (A-I)} sea suficiente (A-II)
Posiblemente una dosis
Metronidazol más gentamicina (B-III)
Una dosis (A-II)
Cistoscopia simple (sin manipulación)

Resección transuretral de próstata

\section{Resección transuretral} de tumor vesical

Inserción/retirada de stent ureteral. Cirugía endourológica ambulatoria

\section{Ureteroscopia con} extracción de cálculos

Litotricia extracorpórea
No recomendada en la cistoscopia simple o urodinamia sin factores de riesgo (D-I)

Recomendada si existen factores

de riesgo ${ }^{\mathrm{b}}$ (B-III)

Recomendada (A-I)

No recomendada (D-III)

Recomendada si factores de riesgo

o tumores de gran tamaño (B-III)

Recomendada en pacientes con

factores de riesgo ${ }^{b}$ (B-III)

Recomendada (C-III)

fundamentalmente en pacientes con factores de riesgo ${ }^{\mathrm{b}}$ (B-III)

No recomendada si no existen factores de riesgo (D-I)

Recomendada en pacientes con

factores de riesgo ${ }^{\mathrm{b}}$ (B-III)
Fosfomicina trometamol o cefuroxima o amoxicilina-ácido clavulánico (A-II)

Fosfomicina trometamol (A-II) o cefuroxima o amoxicilinaácido clavulánico (A-III)

Fosfomicina trometamol (A-II) o cefuroxima o amoxicilinaácido clavulánico (A-III)

Fosfomicina trometamol (A-II) o cefuroxima o amoxicilinaácido clavulánico (A-III)

En pacientes con infección previa o colonización de catéteres es preciso adaptar la profilaxis antibiótica en base a urocultivos previos (B-II)

Fosfomicina trometamol (A-II) o cefuroxima o amoxicilinaácido clavulánico (A-III)

En pacientes con infección previa o colonización de catéteres es preciso adaptar la profilaxis antibiótica en base a urocultivos previos (B-II)

Fosfomicina trometamol (A-II) o cefuroxima o amoxicilinaácido clavulánico (A-III)

En pacientes con infección previa o colonización de catéteres es preciso adaptar la profilaxis antibiótica en base a urocultivos previos (B-II)
Antimicrobiano alternativo si alergia a betalactámicos

Duración Fosfomicina trometamol

Una dosis (A-I) (A-I)

Fosfomicina-trometamol (A-II) o gentamicina (A-III)

Fosfomicina-trometamol (A-II) o gentamicina (A-III)

Fosfomicina-trometamol (A-II) o gentamicina (A-III)

\section{Una dosis (A-I)}

Una dosis (A-I)

Una dosis (A-I)

Fosfomicina-trometamol (A-II) o gentamicina (A-III)

Una dosis (A-I)

Fosfomicina-trometamol (A-II) o gentamicina (A-III) 
Tabla 1 (Continuación)

Cirugía urológica

Indicación

Antimicrobiano recomendado

Antimicrobiano

alternativo si alergia

a betalactámicos

Nefrectomía abierta

No recomendada (D-II), excepto si se

Cefuroxima o amoxicilina-ácido clavulánico (A-III)

Gentamicina (A-III)

Una dosis (A-I)

o laparoscópica

considera cirugía limpia contaminada

o pacientes de alto riesgo ${ }^{\mathrm{b}}$ (B-II)

Nefrolitotomía

Recomendada (A-II)

percutánea

Prostatectomía simple

(vía abdominal y

laparoscópica)

Cistectomía radical con

entrada en el tracto

intestinal. Derivaciones

urinarias

Biopsia transrectal

de próstata

Recomendada (A-II)

Recomendada (A-II)

En pacientes con infección previa o colonización de catéteres

es preciso adaptar la profilaxis antibiótica en base a urocultivos previos (B-II)

Cefuroxima o amoxicilina-ácido clavulánico (A-II)

En pacientes con antecedentes de ITU por BGN productores

de BLEE es preciso adaptar la profilaxis antibiótica (A-III)

Cefuroxima o amoxicilina-ácido clavulánico (A-III)

Gentamicina (A-III)

Una dosis (A-I)

Gentamicina más

vancomicina (B-III)

Una dosis (A-I)

Cefuroxima más metronidazol o amoxicilina-ácido clavulánico

(A-II) (añadir gentamicina en caso de alta prevalencia de BGN resistentes) (B-III)

Recomendada (A-I)

Fosfomicina trometamol (A-I) o cefuroxima o amoxicilinaácido clavulánico oral antes del procedimiento (A-II) (añadir gentamicina si existe una alta prevalencia de BGN resistentes (B-III)

Si existen antecedentes de ITU por microorganismos

multirresistentes es recomendable la profilaxis dirigida (A-II)

Gentamicina más

metronidazol (B-III)

Una dosis (A-II)

Fosfomicina trometamol

(A-I) o gentamicina (A-III)

Una dosis $1-3 \mathrm{~h}$

antes del

procedimiento

(A-I)

No recomendada (D-II) fimosis y otras cirugías

de pene sin

implantación de

prótesis, biopsia renal

abierta.

Prótesis de pene

Cirugía ginecológica

Recomendada (A-III)

Indicación

Cefalosporina de $1 .^{\mathrm{a}}$ o $2 .^{\mathrm{a}}$ generación (A-III)

Antimicrobiano recomendado

Gentamicina más

vancomicina (B-III)

Antimicrobiano alternativo si

alergia a betalactámicos

\section{Cesárea}

Recomendada en la cesárea electiva y

urgente (A-I)

En cesárea electiva cefazolina (A-I)

En cesárea no electiva: cefazolina más

azitromicina (A-I)

Histerectomía

Recomendada en la histerectomía abdominal y vaginal (A-I)

Cefazolina o cefoxitina o amoxicilina-ácido clavulánico (A-I) Clindamicina más gentamicina (B-III)

Clindamicina más gentamicina (B-III) o vancomicina más gentamicina (B-III)

Doxiciclina $100 \mathrm{mg}$ vía oral/2 $\mathrm{h}$ antes intravenosa antes del procedimiento (A-I) azitromicina $1 \mathrm{~g}$ vía oral o intravenosa más

Recomendada en el aborto quirúrgico inducido del primer trimestre (A-I) y del 2 . trimestre o en legrados puerperales (A-III)

No recomendado en el aborto médico (D-III)

Reparación de desgarros Recomendada en la cirugía de desgarros

vaginales posparto vaginales posparto (II//V) (A-I)

\section{de trompas}

borto inducido o

legrados puerperales
Cefoxitina o amoxicilina-ácido clavulánico (A-I) Clindamicina más gentamicina (B-III) Una dosis (A-I) 


\begin{tabular}{|c|c|c|c|c|}
\hline Trasplantes & Indicación & Antimicrobiano recomendado & $\begin{array}{l}\text { Antimicrobiano alternativo si } \\
\text { alergia a betalactámicos }\end{array}$ & Duración \\
\hline Trasplante de riñón & Recomendada (A-II) & $\begin{array}{l}\text { Cefazolina (A-II), considerar añadir gentamicina si alta prevalencia de } \\
\text { BGN resistentes (B-III) }\end{array}$ & $\begin{array}{l}\text { Vancomicina más gentamicina según } \\
\text { la consideración anterior (B-III) }\end{array}$ & Una dosis (A-II) \\
\hline $\begin{array}{l}\text { Trasplante de Páncreas- } \\
\text { combinado páncreas/ } \\
\text { riñón }\end{array}$ & Recomendada (A-II) & $\begin{array}{l}\text { Cefazolina (A-II), o amoxicilina-ácido clavulánico (B-II) (por la frecuente } \\
\text { implicación de enterococos) } \\
\text { Considerar añadir un aminoglucósido según la epidemiología local o la } \\
\text { colonización previa por microorganismos multirresistentes (B-III) } \\
\text { Considerar añadir fluconazol si existe un riesgo alto de infección por } \\
\text { Candida spp (drenaje entérico, trombosis vascular, pancreatitis por } \\
\text { reperfusión) (C-III) }\end{array}$ & Vancomicina más gentamicina (B-III) & $\begin{array}{l}\text { Una dosis (A-II) } \\
\text { Dosis intraoperatorias } \\
\text { adicionales si se prolonga } \\
\text { la cirugía o hay grandes } \\
\text { pérdidas sanguíneas (B-II) }\end{array}$ \\
\hline Trasplante de hígado & Recomendada (A-II) & $\begin{array}{l}\text { Amoxicilina-ácido clavulánico (A-III) } \\
\text { Considerar añadir un aminoglucósido según la epidemiología o la } \\
\text { colonización previa por microorganismos multirresistentes (B-III) } \\
\text { Individualizar la profilaxis según la colonización o la infección previa al } \\
\text { transplante (B-III) }\end{array}$ & Vancomicina más gentamicina (B-III) & $\begin{array}{l}\leq 24 \mathrm{~h} \text { (A-II) } \\
\text { Considerar dosis } \\
\text { intraoperatorias } \\
\text { adicionales si se prolonga } \\
\text { la cirugía o hay grandes } \\
\text { pérdidas sanguíneas (B-II) }\end{array}$ \\
\hline $\begin{array}{l}\text { Trasplante de intestino } \\
\text { delgado }\end{array}$ & Recomendada (A-II) & $\begin{array}{l}\text { Si no existen factores de riesgo de infección por microorganismos } \\
\text { multirresistentes: amoxicilina-ácido clavulánico más gentamicina (A-III) } \\
\text { Considerar ajustar la profilaxis a la microbiología previa, añadir } \\
\text { fluconazol si existen factores de riesgo de Candida spp (B-III) }\end{array}$ & $\begin{array}{l}\text { Vancomicina más gentamicina más } \\
\text { metronidazol (B-III) }\end{array}$ & $\begin{array}{l}\leq 24 \mathrm{~h} \text { (A-II) } \\
\text { Considerar dosis } \\
\text { intraoperatorias } \\
\text { adicionales si se prolonga } \\
\text { la cirugía o hay grandes } \\
\text { pérdidas sanguíneas } \\
\text { (A-III) }\end{array}$ \\
\hline Trasplante de corazón & Recomendada (A-II) & $\begin{array}{l}\text { Cefazolina (A-II) } \\
\text { Aquellos pacientes diagnosticados de una infección asociada a un } \\
\text { dispositivo de asistencia ventricular y que vayan a ser sometidos a un } \\
\text { trasplante cardiaco deberían recibir profilaxis que incluya al } \\
\text { microorganismo implicado (B-III) }\end{array}$ & $\begin{array}{l}\text { Vancomicina. Considerar añadir } \\
\text { gentamicina según epidemiología } \\
\text { local y el riesgo de infección por BGN } \\
\text { (B-III) }\end{array}$ & Una dosis (A-II) \\
\hline $\begin{array}{l}\text { Trasplante combinado } \\
\text { corazón-pulmón }\end{array}$ & Recomendada (A-II) & $\begin{array}{l}\text { Cefazolina (A-II). Este régimen debe modificarse en aquellos pacientes } \\
\text { con cultivos previos o con cultivos del donante positivos (B-III) } \\
\text { Aquellos pacientes diagnosticados de una infección asociada a un } \\
\text { dispositivo de asistencia ventricular y que vayan a ser sometidos a un } \\
\text { trasplante corazón-pulmón deberían recibir profilaxis que incluya al } \\
\text { microorganismo implicado (B-III). } \\
\text { La profilaxis puede incluir en algunas ocasiones antifúngicos con } \\
\text { actividad frente a Candida spp. o Aspergillus spp. en determinados } \\
\text { pacientes (fibrosis quística, donante o receptor colonizados pretrasplante) } \\
\text { (B-III) }\end{array}$ & $\begin{array}{l}\text { Vancomicina, considerar añadir } \\
\text { gentamicina según la epidemiología } \\
\text { local y el riesgo de infección por BGN } \\
\text { (B-III) }\end{array}$ & $\begin{array}{l}\leq 24 \mathrm{~h} \text { (A-II) } \\
\text { No mantener la profilaxis } \\
\text { hasta la retirada de los } \\
\text { drenajes } \\
\text { Si fibrosis quística } \\
\text { tratamiento al menos } 7 \\
\text { días, con cobertura frente } \\
\text { a los microorganismos } \\
\text { aislados pretrasplante } \\
\text { (B-II) }\end{array}$ \\
\hline $\begin{array}{l}\text { BLEE: enterobacterias de } \\
\text { cadera; PTR: prótesis totá } \\
\text { a Cirugía limpia, duració } \\
\text { gravedad. } \\
\text { b Edad avanzada, anoma } \\
\text { exógeno colonizado, infe }\end{array}$ & $\begin{array}{l}2 \mathrm{~h} \text {, no material pr } \\
\text { anatómicas del trac } \\
\mathrm{n} \text { a distancia y hos }\end{array}$ & $\begin{array}{l}\text { o, edad < } 65 \text { años, no comorbilidades ni obesidad, no transfusión, no ex } \\
\text { nario, situación nutricional deficiente, tabaquismo, uso crónico de cortico } \\
\text { zación prolongada. }\end{array}$ & roides, inmunodeficiencias, catéteres & $\begin{array}{l}\text { lina; PTC: prótesis total de } \\
\text { llo de la ILQ no implicaría } \\
\text { ernos, material endógeno o }\end{array}$ \\
\hline
\end{tabular}


Cirugía a través de senos paranasales o faringe. La utilización de PA ultracorta parece eficaz, segura y barata para la prevención de meningitis tras cirugía transesfenoidal.

\section{Cirugía de cabeza y cuello}

Cirugía limpia: cirugía de glándulas salivares, tiroidectomía, paratiroidectomía, vaciamiento ganglionar, sin entrada en mucosas oral, respiratoria o digestiva. La administración de PA no reduce la ILQ en pacientes sometidos a cirugía limpia de cabeza y cuello. Cuando va acompañada de disección ganglionar cervical, la extensa exposición de los tejidos puede incrementar el riesgo de infección, por lo que puede considerarse la profilaxis en pacientes con linfadenectomías extensas o cirugía cervical con resección multivisceral.

Cirugía limpia-contaminada: amigdalectomía, adenoidectomía, laringuectomía, traqueotomía y cualquier otra cirugía en la que se realice incisión de la mucosa faringo-laríngea. La PA es recomendada en cirugía de cabeza y cuello limpia-contaminada, excepto en adenoidectomía y amigdalectomía. Se aconseja también en cirugía oncológica por neoplasias de cabeza y cuello, donde se ha observado una elevada tasa de infección quirúrgica.

Cirugía de senos paranasales y de oído medio. No se recomienda la PA en la cirugía endoscópica de los senos paranasales ni en cirugía limpia otológica. En cambio, se aconseja la aplicación tópica de antibiótico después de timpanoplastia y la PA en cirugía limpia contaminada y contaminada. La PA en el implante coclear es controvertido, está indicada por inferencia de la cirugía limpia con colocación de implante.

Cirugía maxilofacial. No se ha demostrado beneficio de la PA en la septoplastia y la rinoplastia simple a pesar de ser una cirugía limpia-contaminada. En la rinoplastia compleja (revisión, prótesis) sí estaría recomendada.

Fracturas maxilofaciales. La información sobre el impacto de la profilaxis en la cirugía de fracturas maxilofaciales es limitada, pero en general se recomienda la PA, sobre todo en la fractura mandibular que requiera reducción abierta.

Procedimientos odontológicos. En extracción dental, implante oral o endodoncia no se recomienda la PA, que solo sería necesaria si existen factores de riesgo (edad, cirugía o infección previa, tabaquismo, enfermedades sistémicas y extracción traumática). La PA está recomendada en la implantación de injerto óseo a través de la cavidad oral.

\section{Cirugía traumatológica y ortopédica}

Los procedimientos quirúrgicos traumatológicos y ortopédicos son mayoritariamente cirugía limpia, con frecuencia acompañadas de implantes y, aunque la incidencia de ILQ es baja, esta complicación puede ser catastrófica, recomendándose PA en la mayoría de ellos. La PA no está recomendada en pacientes sometidos a cirugía limpia ortopédica que no se acompaña de instrumentación, incluida la artroscopia sin ligamentoplastia ni en la retirada de implante ortopédico.

Reducción de fractura cerrada con material osteosíntesis. Los pacientes con fracturas de cadera suelen ser ancianos, con comorbilidades y muchas veces institucionalizados. En ellos, con frecuencia se detectan infecciones por SARM o polimicrobianas con la implicación de bacilos gramnegativos. Se recomienda la PA en la reducción de fractura cerrada con material osteosíntesis, con cobertura para SARM si hay factores de riesgo.

Intervención de fractura abierta. La PA debe administrase lo más cercano al momento de la fractura. En las fracturas grados I y II de Gustilo se recomienda mantener hasta $24 \mathrm{~h}$ del desbridamiento, y en las de grado IIIA un máximo de $72 \mathrm{~h}$ o hasta la cobertura de los tejidos blandos.

Artroplastias (PTC, PTR, megaprótesis tumorales, primarias y revisión). La PA en monodosis demuestra un beneficio. En las artroplastias de revisión por infección protésica, se incrementa el riesgo de infección y de infecciones por microorganismos resistentes, por lo que se recomienda PA de amplio espectro que cubra el microorganismo causante de la infección inicial.

Laminectomías y discectomías sin/con instrumentación. La PA está recomendada.

Amputación de miembros. La PA está recomendada en la amputación de extremidades inferiores al menos $24 \mathrm{~h}$.

\section{Cirugía torácica}

Cirugía torácica mayor y mínimamente invasiva. Se recomienda la PA en cirugía torácica mayor y cirugía mínimamente invasiva (videotoracoscopia, mediastinoscopia).

Colocación de tubo torácico, traumatismo penetrante. No está recomendada en la colocación electiva de tubos torácicos, pero sí en caso de traumatismo torácico penetrante.

\section{Cirugía esófago-gastro-duodenal}

Con rotura de mucosa (esofaguectomía, gastrectomía, duodenopancreatectomía cefálica). Se recomienda la PA.

Sin rotura de mucosa (cirugía de reflujo gastroesofágico, achalasia, vagotomía). La PA solo está recomendada en pacientes de alto riesgo (obesidad mórbida, obstrucción intestinal, hipoclorhidria, hemorragia gástrica, neoplasia, perforación, inmunosupresión o clasificación ASA > 3).

Implantación de PEG (gastrotomía percutánea endoscópica). Recomendada la PA.

Cirugía bariátrica. Por extrapolación de procedimientos similares la PA está recomendada, ajustada al peso.

\section{Cirugía de intestino delgado}

Está recomendada la PA al ser una cirugía limpia contaminada, por inferencia de otras localizaciones sobre todo de la colorrectal.

\section{Esplenectomía}

La esplenectomía no traumática no tiene indicación de PA, sí la tienen la esplenectomía traumática o la electiva en pacientes de alto riesgo (inmudeprimidos o bajo tratamiento inmunosupresor, ancianos con enfermedades debilitantes, cirugía $>120$ min o pérdida sanguínea elevada).

\section{Traumatismo penetrante de abdomen}

Indicada la PA preoperatoria con cobertura frente a aerobios y anaerobios, manteniéndola no más de 24 h si existe lesión de 
víscera hueca. En caso de shock hemorrágico la dosis debe ser 2-3 veces mayor y repetida después de cada 10 unidades de sangre transfundida.

\section{Apendicectomía}

Se recomienda la PA en apendicectomía no complicada, pero la complicada es tributaria de tratamiento antibiótico (absceso, plastrón, peritonitis difusa o perforación).

\section{Cirugía colorrectal}

La cirugía colorrectal electiva es limpia-contaminada y presenta las tasas de ILQ más altas de la cirugía gastrointestinal. La PA electiva disminuye la tasa de ILQ. Se recomienda la preparación mecánica del colon asociada a antibióticos orales en cirugía colorrectal electiva.

\section{Cirugía hepato-bilio-pancreática}

Colecistectomía y cirugía biliar. En la colecistectomía laparoscópica en pacientes de bajo riesgo la PA no está recomendada, pero debería considerarse en colecistectomía abierta y todas las situaciones de alto riesgo: intervención de emergencia, inmunodepresión, diabetes, embarazo, edad $>70$ años, clasificación ASA $\geq 3$, episodio de cólico biliar dentro de los 30 días previos al procedimiento, ictericia, coledocolitiasis, colangitis, cirugía biliar previa, colecistitis aguda en los últimos 6 meses, pancreatitis litiásica y prótesis en vía biliar y antibioterapia en el último mes.

Cirugía hepática. La PA no está recomendada en hepatectomía simple, pero sí en hepatectomía mayor (incluye resección biliar extrahepática). En caso de cultivo biliar previo se aconsejar ajustar la PA a los mismos.

Cirugía pancreática. La PA está indicada por inferencia de la cirugía biliar. Cuando existen cultivos previos de la bilis se puede ajustar la antibioterapia al patrón de sensibilidad de los microorganismos aislados.

\section{Cirugía peritoneal avanzada-peritonectomía}

No existen estudios prospectivos, pero dado el abordaje radical de la carcinomatosis peritoneal parece indicado administrar PA adaptada al procedimiento que se realice (esplenectomía, pancreatectomía, colecistectomía, apendicectomía, linfadenectomías y resecciones viscerales gastrointestinales o colorrectales).

\section{Cirugía urológica}

La cirugía urológica, abierta o laparoscópica, está clasificada como limpia-contaminada y es tributaria de PA. La cistoscopia simple, la cirugía endourológica ambulatoria y la litotricia extracorpórea son procedimientos invasivos no tributarios de profilaxis, salvo que existan factores de riesgo que alteren la respuesta frente a las infecciones: edad avanzada, anomalías anatómicas del tracto urinario, situación nutricional deficiente, tabaquismo, uso crónico de corticosteroides, inmunodeficiencias, catéteres externos, material endógeno o exógeno colonizado, infección distante u hospitalización prolongada. La recomendación de profilaxis en procedimientos urológicos abiertos o laparoscópicos como la nefrectomía, se basa en las recomendaciones generales de profilaxis según el grado de contaminación. Cuando está indicada la PA, una dosis preoperatoria previene la infección, el tipo de antibiótico ha de adaptarse al patrón de sensibilidad local y programas de optimización de antimicrobianos del centro.

Resección transuretral de próstata. Los estudios aleatorizados aconsejan la administración de PA.

Resección transuretral de tumor vesical. La PA no está recomendada a no ser que existan factores de riesgo o se trate de tumores de gran tamaño.

Ureteroscopia con extracción de cálculos y litotricia extracorpórea. Se sugiere que la PA se limite a la litiasis ureteral no complicada en pacientes con factores de riesgo o en pacientes con litiasis ureteral complicada o impactada.

Nefrectomía abierta o laparoscópica. Al tratarse de cirugía limpia, no está indicada la PA, excepto en pacientes de alto riesgo.

Nefrolitotomía percutánea. La nefrolitotomía percutánea con orina estéril es cirugía limpia-contaminada y tributario de PA.

Prostatectomía simple (vía abdominal y laparoscópica). Es cirugía limpia-contaminada y tiene indicación de PA.

Cistectomía radical con entrada en el tracto intestinal. Derivaciones urinarias. Es cirugía limpia-contaminada con resección intestinal, con alta tasa de infección postquirúrgica, por lo que se recomienda PA.

Biopsia transrectal de próstata. Dos metaanálisis han demostrado el beneficio de la PA en monodosis.

\section{Cirugía ginecológica}

Cesárea. La PA está recomendada en la cesárea urgente y electiva. Varios estudios y metaanálisis y las guías más recientes apoyan su administración antes de la incisión.

Histerectomía. La histerectomía vaginal y abdominal, se consideran cirugía limpia-contaminada tributaria de PA.

Anexectomía y ligadura de trompas. Se consideran cirugía limpia, con bajo riesgo de infección, que no precisa PA.

Aborto inducido o legrados puerperales. La PA está recomendada en el aborto quirúrgico del primer y segundo trimestre o legrados puerperales, no está recomendada en el aborto médico.

Reparación de desgarros vaginales posparto. Se recomienda administrar PA en los desgarros vaginales posparto grados III y IV (los que afectan a ano o recto, respectivamente).

\section{Trasplantes}

Los trasplantes de riñón, páncreas, hígado, intestino delgado, corazón y pulmón se asocian a una alta tasa de ILQ a veces con la pérdida del injerto, por lo que se recomienda la PA. No existen estudios comparativos de calidad que evalúen su eficacia ni la mejor pauta de antibiótico. Las recomendaciones se basan en estudios observacionales y en la inferencia de otras cirugías, adaptadas a la microbiología previa.

\section{Conclusiones}

La PA es una de las principales medidas de prevención de la ILQ. Su objetivo es asegurar una alta concentración 
de antibiótico en los tejidos que van a ser intervenidos antes del inicio y durante el procedimiento. En general, está recomendada cuando las probabilidades de infección postoperatoria son elevadas o cuando sus consecuencias son potencialmente graves. Ello incluye, como mínimo, las intervenciones clasificadas como limpia-contaminada y contaminada y la cirugía limpia con implantación de material protésico.

Los antibióticos seleccionados deben ser activos frente a los microorganismos aislados con más frecuencia en cada tipo de procedimiento quirúrgico y suelen ser cefalosporinas de primera o segunda generación. Los fármacos deben administrarse por vía intravenosa en los 120 min previos a la incisión quirúrgica, a dosis máximas terapéuticas, con modificación en los pacientes obesos.

Para la mayoría de los procedimientos se recomienda una dosis única preoperatoria y nunca debe prolongarse más allá de las primeras $24 \mathrm{~h}$ del postoperatorio. La redosificación intraoperatoria es más importante que la administración de dosis postoperatorias cuando la herida está ya cerrada y está indicada cuando el procedimiento quirúrgico excede 2 veces la semivida del antibiótico.

Todo protocolo de PA en cirugía debería incluir el registro de los indicadores de su cumplimiento, el análisis de los resultados y el feedback de los mismos a los miembros de los equipos quirúrgicos.

\section{Financiación}

La presente investigación ha sido patrocinada por la SEIMC y no ha recibido ayudas específicas provenientes de agencias del sector público, sector comercial u otras entidades sin ánimo de lucro.

\section{Conflicto de intereses}

Los autores declaran no tener ningún conflicto de intereses relacionado con este manuscrito.

\section{Agradecimientos}

Los autores agradecen a Antonio Gutierrez-Pizarraya su contribución en el soporte bibliográfico de este documento, y a la SEIMC y la AEC por depositar su confianza para la elaboración del manuscrito.

\section{Anexo. Material adicional}

Se puede consultar material adicional a este artículo en su versión electrónica disponible en doi:10.1016/j.ciresp.2020.03.022. 